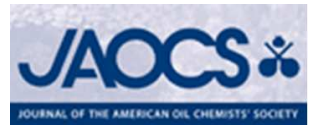

\title{
Controlling argan seed quality by NIR
}

\begin{tabular}{|r|l|}
\hline Journal: & Journal of the American Oil Chemists Society \\
\hline Manuscript ID: & JAOCS-14-0306.R2 \\
\hline Danuscript Type: & Original Article \\
\hline Complete List of Authors: & $\begin{array}{l}\text { Guinda, Angeles; Instituto de la Grasa (CSIC), Department of Food Quality } \\
\text { and Characterization } \\
\text { Rada, Mirela; Instituto de la Grasa (CSIC), Department of Food Quality and } \\
\text { Characterization } \\
\text { Benaissa, Mohamed; Hassan II Aîn Chock University, Faculty of Sciences } \\
\text { Ourrach, Ibtissam; Hassan II Aîn Chock University, Faculty of Sciences } \\
\text { Cayuela, José; Instituto de la Grasa (CSIC), Department of Food Quality } \\
\text { and Characterization }\end{array}$ \\
\hline Keywords: & Oilseeds, Lipid Chemistry / Lipid Analysis \\
\hline \multicolumn{2}{|c}{} \\
\hline
\end{tabular}

SCHOLARONE

Manuscripts 


\section{Controlling argan seed quality by NIR}

Ángeles Guinda ${ }^{1}$, Mirela Rada ${ }^{* 1}$, Mohamed Benaissa ${ }^{2}$, Ibtissam Ourrach ${ }^{2}$, José Antonio Cayuela ${ }^{1}$

${ }^{1}$ Department of Food Quality and Characterization. Instituto de la Grasa-CSIC. Campus of Pablo de Olavide University, Building 46 Utrera Road, km 1. 41013Seville-Spain.

${ }^{2}$ Faculty of Sciences, Universite Hassan II, AinChock-BP 5366 Maarif-CasablancaMorocco.

${ }^{*}$ Corresponding author: Mirela Rada email:mrada@ig.csic.es

Phone: ++34 954611550 (ext. 209)

Fax: $\quad++34954616790$ 


\begin{abstract}
The suitability of using Vis/NIR, as a rapid and non-destructive technique for monitoring the quality of argan seeds (Argania spinosa Skeels) was studied. The analyzed parameters were the fatty acid composition of argan seed oil, seed moisture content, seed oil content and oil stability index (OSI). The ratio between major unsaturated and saturated fatty acids (U/S) during the oxidation assay at constant temperature was studied. Values from infrared drying were used as laboratory reference for the moisture. Argan seed oil content was determined by Soxhlet extraction. A fatty acid analysis was carried out by gas chromatography and OSI was determined by the Rancimat test. Predictive models of argan seed moisture, ratio U/S and OSI showed good accuracy. Therefore, Vis/NIR measurements can be used for controlling several argan seed quality parameters. This procedure might be of interest to the argan oil industry, which is currently in the process of modernization and expansion.
\end{abstract}

Key words: argan, fatty acids, moisture, Vis/NIR, oil seeds. 


\section{Introduction}

Argan oil is characterized by a high level of monounsaturated and di-unsaturated fatty acids, oleic and linoleic acids, respectively. Fats rich in unsaturated fatty acids, such as argan oil, are largely recommended for their healthy properties. Moreover, argan oil is rich in tocopherols that have an important antioxidant activity. In addition, argan oil is rich in phytosterol, whose incorporation into the diet is believed to offer cancer protection [1].

The Argan tree (Argania spinosa L. Skeels), belonging to the Sapotaceae family, presents an irreplaceable ecological function in Morocco, protecting the soil against erosion, maintaining soil fertility and guaranteeing most of the dietary needs of small scale farmers [2]. Argan is primarily used for its oil, but it is also utilized as fuel-wood and food for domestic goats and camels. In addition, the Moroccan folk medicine uses argan oil-based preparations for their hepatoprotective, hypocholesterolemic, and anti-atherosclerotic effects, and also in the treatment of different diseases such as rheumatism and dermatological and articular pathologies [3, 4]. The extraction of the oil contained in the seeds of argan has been a traditional process for centuries; however, a modernization is in progress which implies a mechanization which can reduce the manual labor and at the same time, facilitate an improvement in the argan oil quality. For this purpose, several cooperatives for the production and commercialization of quality certified argan oil have been initiated in South-West Morocco [5].

An important characteristic of the Argan tree is the presence of the group of triterpenoid saponins, generically arganine [6] with an astringent, bitter taste, which needs to be removed by seed roasting in the edible argan oil production. Argan kernel roasting also deserves attention because it induces a pleasant hazelnut aroma and taste that are transferred, during extraction, to the oil [7]. However, for cosmetic uses, seed roasting is not required. Therefore, two kinds of oils are produced, depending on the oil use and according to the treatment applied to the seeds. Raw material quality, roasting and storage conditions are important steps to make high quality edible seed oil $[8,9]$. Oil plant processing influences fatty acid composition and its oxidative stability, among other parameters. The inappropriate toasting of argan seed or it storage conditions can induce changes in the relationship between unsaturated and saturated fatty acids (U/S), as well as in the argan oil oxidation induction time, due to a higher incidence of oxidation of the unsaturated fraction [10].

The oil's oxidative stability was measured years ago by the active oxygen method (AOM), expressed in hours, according to the AOCS official method Cd. 1-57 [11]. Nowadays, AOM has been replaced by the oil stability index (OSI) method Cd 12b-92 with the Rancimat apparatus [11]. The OSI determination presents some difficulties, particularly the long period of time for analysis 
and the necessary instrument cleaning. For these reasons, the applicability of new methods has been investigated [12].

However, the information reported about directly measuring the oxidative state of intact oil seeds is practically nonexistent. Therefore, Visible and Near Infrared Spectroscopy measurements can be useful for controlling some argan seed quality parameters, and in this way facilitating a rapid and nondestructive technique in the semi-industrial process of virgin argan oil extraction. Vis/NIR is successfully used for the routine analysis of different quality parameters such as moisture, oil or protein contents in several grains and oilseeds [13, 14]. Siemens and Daun have reported that it is possible to determine the content of fatty acids (FA) in oil seeds by Vis/NIR spectroscopy [15].

However, the feasibility of the technique may depend on the morphological and structural features of the specific seeds. The argan seed shape is flat and its size is relatively large (length 10-23 mm and width 5-12 mm). Results of predictive calibrations of quality parameters of the intact argan seed obtained from their Vis/NIR spectra have never been reported until now.

A technique to determine OSI in vegetable oils from a non-destructive measurement of intact seeds can be very useful, particularly in the case of argan edible oil, in which the seed roasting may affect oil quality. In this work, the suitability of determining the quality parameters fatty acid composition, $\mathrm{U} / \mathrm{S}$ ratio, OSI and seed moisture in intact argan seeds by Vis/NIR spectroscopy was investigated. To this end, we assessed the suitability of two spectrometers. Several of the predicted parameters are reported for the first time.

\section{Materials and methods}

Plant material

Samples of argan (A. spinosa) fruits were supplied by the industrial plant Arganoil Company Ltd. Morocco. The argan seed storage conditions were: temperature $5{ }^{\circ} \mathrm{C}$ and $\mathrm{N}_{2}$ atmosphere until analysis. Under these conditions, no mold development was observed, free fatty acid formation was minimized and the oil oxidation was prevented. 187 samples of argan seeds were analyzed for major FA, 65 for oil stability index, 67 for oil content and 106 for moisture content.

\section{Oxidation assay}

Argan seeds were oven heated at constant temperature $\left(80^{\circ} \mathrm{C}\right)$, and samplings $(15 \mathrm{~g})$ were carried out at 2, 4, 6, 12 and 24 days. Vis/NIR and NIR spectra were acquired after each sampling from the forced oxidation assay employing $5 \mathrm{~g}$. This non-destructive technique allowed for reutilization of the seeds for oil Soxhlet extraction. The extracted argan oil was used for the analysis of fatty acid methyl esters and determination of the oxidation induction time. 


\section{Spectral acquisition}

The spectral acquisition of samples was performed using Labspec Pro (Analytical Spectral Devices Inc., Boulder) and Luminar 5030 (Brimrose Corp., Maryland) portable spectrophotometers. Labspec Pro is a Visible/Near Infrared (Vis/NIR) spectrometer equipped with three detectors. The detector for the visible range $(350-1000 \mathrm{~nm})$ is a fixed reflective holographic diode array with sensitivity at 512 pixels. A holographic fast scanner InGaAs detector cooled at $-25{ }^{\circ} \mathrm{C}$ covers the wavelength range from 1000 to1800 $\mathrm{nm}$. The same device before mentioned coupled with a highorder blocking filter operates for the 1800-2500 nm interval. The instrument is equipped with internal shutters and automatic offset correction. The scanning speed was 100 milliseconds. The spectra acquisition was performed using the 'Turn Table' accessory of the spectrometer with standard SMA 905 fiber optic connectors. The whole spectrum $(350-2500 \mathrm{~nm})$ reflected from the entire surface of the argan seed sample was acquired at $1 \mathrm{~nm}$ intervals for each spectral variable. Indico Pro software (Analytical Spectral Devices Inc., Boulder) was employed for data acquisition. Luminar is an Acousto-Optic Tunable Filter-Near Infrared AOTF NIR spectrophotometer, equipped with a reflectance post dispersive optical configuration and InGaAs (1100-2300 nm) detector. The reference spectrum is automatically taken by the instrument, just as a UV-VIS spectrophotometer dual beam; the beam is divided before leaving and a small portion is sent to a second detector that gives the reference. The scanning speed in Luminar is 60 milliseconds. The hand-held and computer units, with a total weight of $5.26 \mathrm{~kg}$, offer good portability. The hand-held unit is equipped with a base for facultative use in the laboratory. The whole spectrum (1100-2300 nm) was acquired at $1 \mathrm{~nm}$ intervals for each spectral variable. The Vis/NIR radiation beam is projected through a frustoconical tip with an exterior of approx. $1 \mathrm{~cm}$ diameter. The spectra acquisition was performed by using the rotary accessory of the spectrometer base, so that the center of the Vis/NIR beam scans the largest possible area of seeds in its circular path. Acquire software (Brimrose Corp., Maryland) was used for signal acquisition. The argan seed samples were approximately 5 grams for the two spectrometers and about 50 spectra were analyzed to obtain the average spectrum of each sample. A stainless steel capsule of $40 \mathrm{~mm}$ diameter and $20 \mathrm{~mm}$ height was used as seed sample container. The samples were introduced separately in hermetic plastic pots and labeled until their reference analysis.

\section{Fatty acid analysis}

Fatty acid methyl esters (FAME) were obtained by a trans-esterification procedure carried out with a cold methanolic solution of potassium hydroxide [16]. This rapid method is applicable to oils with a free FA content of less than $3.3 \%$. Briefly, in a $5 \mathrm{~mL}$ screw-top test tube, approximately $0.1 \mathrm{~g}$ of the oil sample is weighed and $2 \mathrm{~mL}$ of heptane is added and shaken. Subsequently, $0.2 \mathrm{~mL}$ of a $2 \mathrm{~N}$ 
methanolic potassium hydroxide solution were then added and was shaken vigorously for $30 \mathrm{~s}$. It was then stratified until the upper solution containing the methyl esters becomes clear. The GC analysis of FAMEs in heptane is carried out using a fused silica capillary column (SP-2380, $60 \mathrm{~m} \mathrm{x}$ $0.25 \mathrm{~mm}$ I.D.) coated with cyanopropyl-silicone $(0.20 \mu \mathrm{m}$ film thickness $)$ and using hydrogen as carrier gas. The initial oven temperature of $160^{\circ} \mathrm{C} / 13 \mathrm{~min}$ was increased at $1.5^{\circ} \mathrm{C} / \mathrm{min}$ until 190 ${ }^{\circ} \mathrm{C}$ and maintained for $5 \mathrm{~min}$. Injector and detector temperatures were $225{ }^{\circ} \mathrm{C}$ and $250{ }^{\circ} \mathrm{C}$, respectively. The primary FA considered were those containing 5\% or more FA methyl esters.

Statistical analysis of the experimental data ANOVA was carried out using the SigmaStat 3.5 statistical pack (Systat Software Inc.). Tukey's test was used to determine specific differences among group means. Differences were considered significant at $\mathrm{P} \leq 0.001$.

\section{Determination of oil stability index}

The OSI, or the oxidation induction time, was determined according to the AOCS Official Method Cd 12b-92 [11]. The air flow was $10 \mathrm{~L} / \mathrm{h}$ and the oil was heated at $100{ }^{\circ} \mathrm{C}$, using the Rancimat apparatus (743 Rancimat; Metrohm Ltd., Herisau, Switzerland). OSI was expressed as h of induction of the complete oxidation of FA.

\section{Moisture content}

The argan seed moisture was determined by gravimetry, considering the difference between fresh and dry weight employing an HG63 Halogen Moisture Analyzer (Mettler Toledo, SAE). Each sample of approximately $5 \mathrm{~g}$ of argan seed was ground in a mechanical mill. The resulting powder was placed in an aluminum sample pan (HA-D90) and dried at a temperature of $100{ }^{\circ} \mathrm{C}$, using the rapid drying program. The end-point was determined when the weight loss in $50 \mathrm{~s}$ was less than 1 mg.

\section{Oil content}

The dry material resulting from the procedure described in the previous section was weighed and the oil content was determined following the American Oil Chemists Society (AOCS) Official methods [17].

\section{Chemometry and calibration procedure}

The predictive models for the parameters analyzed were built using 4/5 of the total corresponding sample number by Partial Least Squares method (PLS) [18]. The remaining samples (1/5) were reserved for external validation. 
Calibrations were obtained using PLS with The Unscrambler (CAMO Software AS, Norway) and the full spectrum was studied. The models principal components (PCs) were fixed after tests using 10 PCs initially. The procedure for spectral variable selection to be included in the models consisted of performing several consecutive cycles, eliminating variables having spectral correlation coefficients with the parameters analyzed closer to zero. Variable selection ended in the last cycle that improved the statistical model $\mathrm{R}$ and $\mathrm{R}_{\mathrm{CV}}$. This selection was made on the regression coefficient graph of The Unscrumbler.which summarizes the relationship between the Vis/NIR wavelengths and the response.These cycles of elimination were conducted by means of the 'mark with rectangle' and the 'recalculate without marked' specific applications of The Unscrambler.

The reflectance data was normalized to its maximum. Standard Normal Variate Transformation (SNV) and Savitzsky-Golay second derivative, with segment width 3 and polynomial order 2 was also tested. Full-cross internal validation (FCV) was used for the models.

\section{Validation procedure}

External validation exercises were carried out using the corresponding models to predict the parameters on completely independent samples. The performance from the PLS procedure was assessed by the ratio of the standard deviation of the reference data from the validation set to the SEP, the residual predictive deviation (RPD). The standard error of performance (SEP) is computed as the standard error of the residuals and expressed in the same unit as the original values. According to Williams and Sobering (1996) [19], RPD should ideally be three or higher for good model performance. SEP and the root mean square error of prediction (RMSEP) were considered. In addition, the coefficient of variation (CV) and the range of each major FA expressed as relative range, in percentage, $(\mathrm{R})$ were also considered for assessing the influence of the statistics in the performance of the models.

\section{Results and Discussion}

Argan seed spectra

An example of argan seed absorbance spectra acquired with the Labspec Vis/NIR spectrometer used is shown in Fig. 1. Absorption shows oscillations from 850 to $1200 \mathrm{~nm}$, falling and rising again to a new peak between 1400 and $1500 \mathrm{~nm}$. Another peak observed between 1700 and 1800 $\mathrm{nm}$ is most probably due to oil and its composition and the other one between 1900 and $2000 \mathrm{~nm}$, which can be attributed to water with oscillating and rising absorption at 2050-2150 nm and at $2300-2500 \mathrm{~nm}$ [20]. The region close to $2180 \mathrm{~nm}$ is related to the protein content, as reported by Velasco et al., in Ethiopian mustard seeds [14]. 
Spectral Variable Analysis

The analysis of wavelength regression coefficients showed different spectral intervals, which have contributed significantly to the positive outcome of the Labspec calibrations (Tab. 1). On the contrary, wavelength intervals with decisive importance were not identified with the Luminar calibrations for the different analyzed parameters.

\section{Reference Analysis}

Table 2 shows the statistical data of the reference analysis of fatty acids and U/S ratio of the argan seed samples from the forced oxidation assay. For the corresponding calibrations and validations all samples (2, 4, 12 and 24 days) were considered together as a simple set. The ranges and standard deviations indicate the diversity of the analyzed parameters integrated in the calibration and validation sets. Oleic acid in particular, which has the smallest statistical range of all the examined parameters (6.04\%), influences the corresponding model as discussed below.

\section{Major fatty acids and U/S ratio}

Calibrations for the prediction of the major FA in intact argan seeds were conducted in the assays performed with Luminar and Labspec. The statistical data of the sample sets used for the major FA calibrations and validations are included in Tab. 2, and the statistical coefficients from the Labspec and the Luminar are included in Tab. 3.

The difference between the wavelengths integrated in the spectrum, which with Luminar 5030 was NIR exclusively; while it was Vis/NIR with Labspec, contribute to the best results achieved with the last one. This has been tested with different calibrations of the Labspec excluding the range of visible wavelengths, whose statistics were worse than that reached using also the visible wavelengths shown in Table 3. As some examples, Rc from stearic acid calibration was 0.96 using Vis, while 0.85 with only NIR. In the case of U/S and OSI calibrations, their Rc were 0.98 and 0.99 when including Vis, as shown in Table 3, while 0.96 and 0.98 with only NIR.

The same above reasons may be the cause why the oleic acid calibration built with Luminar 5030 did not reach sufficient accuracy for its practical use, so external validation data are not shown.

The evolution of the major FA in the forced oxidation assay of intact argan seeds is shown in Table 4. Considering these data, we observed that the treatment of argan seeds at $80{ }^{\circ} \mathrm{C}$ for 24 days induces the degradation of linoleic acid due to its unsaturation degree. It presented significant differences after statistical comparison $(\mathrm{P} \leq 0.001)$ over all the different steps of the oxidation assay which decreased by up to $13 \%$ in 24 days. Consequently, the relative percentage ratios of U/S are 
reduced, as expressed by the data in the Tab. 4. The greatest reduction was observed in 24 day samples (20\%).

Figure 3 shows the prediction graphs from the forced oxidation assay of argan seeds, corresponding to the external validation exercises for the oleic (a), linoleic (b), palmitic (c) and stearic (d) acids with the Labspec. The RPD coefficients from the external validation exercises were particularly good for the prediction of linoleic, palmitic and for the relation U/S (Fig. 3 (e). The results demonstrate the high predictive capability of the models, particularly showing the possibility to determine the ratio between major unsaturated and saturated fatty acids (U/S) in intact argan seeds with good accuracy.

Interestingly, oleic acid, the most abundant FA in argan seed oil, provided weaker predictive performance than the remaining major FA. This is most probably due to the narrow range shown by its statistical values and consequently lower coefficient of variance. In fact, high correlations $(0.91)$ between RPD values resulting from the validation exercises for each FA with the R values were observed. The correlation between RPD and CV values was lower (0.35), thus showing the important influence of the range of variation of a parameter on its suitability for a good PLS model for Vis/NIR prediction.

The statistical coefficients of the developed model for the OSI prediction are included in Table 4. The prediction in the external validation exercise carried out using the OSI model on 16 independent samples provided an RPD of 4.88, which reveals the high accuracy performance of the model (Fig. 3 (f). A calibration test was also carried out employing all available samples (65), reaching a cross validation coefficient of 0.97 and SEC 2.32, which indicates adequate goodness of the calibration according to the PLS procedure validation (data not shown). This work reports the ability to predict the OSI of argan seed oil through the Vis/NIR spectrum of the intact seeds for the first time.

\section{Argan Seed Moisture}

The statistics from argan seed moisture in the calibration and validation sets conducted with the Luminar 5030 spectrometer are included in Tab. 5. As can be observed, seed moisture variation is higher than the oil content variation. The calibration results for seed moisture prediction, with SEC 0.24 and $\mathrm{R}^{2} 0.83$ using the Luminar are shown in Fig. 2 (a). Its statistical coefficients are included in table 5. Normalized absorbance data mean, smoothed by mean filter and SNV were the data pretreatments applied for this model. An external validation was carried out using $1 / 5$ of the total number of samples, reserved for this aim, and therefore not included in the calibration set. The RPD ratio in the prediction exercise was 2.13. The statistical coefficients from the calibration test for moisture prediction using the Luminar spectrometer, (85 samples), are included in Table 5. The low 
moisture content of argan seeds, which characterizes this parameter as secondary, may be the reason why the accuracy achieved is not very remarkable in this case.

\section{Argan Seed Oil Content}

As for the proceeding parameter, absorbance data mean normalization followed by mean filter smoothing and SNV were the data pre-treatments with best yields. The Fig. 2 (b) shows the dispersion plot of the prediction given by this calibration with the Luminar. The SEC for this calibration was 2.73 and $\mathrm{R}^{2} 0.83$. The sizes of the calibration and validation sets used for this parameter were smaller to those of argan seed moisture (Tab. 5). An external validation exercise was carried out using this calibration, resulting in RMSEP 2.62 and RPD 2.44. The calibration test was carried out with the Luminar and the statistical coefficients are included in Table 5. The results of the tests showed good predictive potential for oil content, encouraging the development of predictive models for the determination of analytical parameters with practical interest in argan seeds.

\section{Conclusions}

The results of this research show that Vis/NIR can be suitable to predict rapidly, nondestructively and with the necessary accuracy, the relationship between unsaturated and saturated major fatty acids in intact argan seeds, and thus facilitating seed quality control. In addition, it has been possible to determine the stability index of argan oil, the composition of major fatty acids and seed oil content. Similarly, although with lower model predictive capacity due to the lower range of variation in the parameter, it was possible to predict the moisture of the seeds. In order to establish viable prediction models employing this technique, appropriate protocols for calibration and validation are required, as well as the assessment of models' robustness. Portability may be an advantage for using the same unit NIR or Vis/NIR at several cooperatives processing argan seeds, which could facilitate the return on investment. It could also facilitate the handling and sorting of argan seeds during different stages of processing. The advantages of a non-destructive and environmentally friendly Vis/NIR technique could be of interest for the argan oil industry, currently undergoing modernization and expansion.

\section{Acknowledgements}

The authors are grateful to the Spanish Agency for the International Cooperation (AECID-MAE) for financing the Project A/019935/08. They also express their gratitude to the industrial plant Argan Oil Company Ltd. Morocco for the argan seed supply. 


\section{References}

[1] Khalloukhi F, Younos C, Soulimani R, Oster T, Charrouf Z, Spiegelhader B, Bartsch H, Owen RW (2003) Consumption of argan oil (Morocco) with its unique profile of fatty acids, tocopherols, squalene, sterols and phenolic compounds should confer valuable cancer chemo preventive effects. Eur J Cancer Prev 12: 67-75

[2] Lybbert T, Magnan N, Aboudrare A (2010) Household and local forest impacts of Morocco's argan oil bonanza. Agric Environment and Development Economics 15: 439464

[3] Bellakhdar J, (1997) La Pharmacopee Marocaine Traditionnelle, Ibis Press, Paris

[4] Moukal A, (2004) L'arganier, argania spinosa L. (Skeels), usage therapeutique, cosmetique et alimentaire. Phytoterapie 2: 135-141

[5] Charrouf Z, Guillaume D, Driouich A (2002) L'arganier, un atout pour le Maroc. Biofutur 220: $54-57$

[6] Charrouf Z, Wieruszeski JM, Fkih-Tetouani S, Leroy Y, Charrouf M, Fournet B (1992) Triterpenoid saponins from Argania spinosa. Phytochemistry 31: 2079-2086

[7] Charrouf Z, El Hamchi H, Mallia S, Licitra G, Guillaume D (2006) Influence of roasting and seed collection on argan oil odorant composition. Nat Prod Commun 1: 399-404

[8] Harhar H, Gharby S, Guillaume D, Charrouf Z (2010) Effect of argan kernel storage conditions on argan oil quality. Eur J Lipid Sci Technol 112: 915-920

[9] Harhar H, Gharby S, Kartah B, El Monfalouti H, Guillaume D, Charrouf Z (2011) Influence of Argan Kernel Roasting-time on Virgin Argan Oil Composition and Oxidative Stability. Plant Foods Hum Nutr 66:163-168

[10] Melton SL (1983) Methodology for following lipid oxidation in muscle foods. Food Technol 37: 105-111

[11] AOCS (1993) Oil stability index In: Firestone D (ed) Official Methods and Recommended Practices of the American Oil Chemists' Society. 3rd edn. AOCS Press, Champaign, methods Cd 12b-92 and Cd 1-57

[12] Kodali D (2005) Oxidative Stability Measurement of High-Stability Oils by Pressure Differential Scanning Calorimeter (PDSC). J Agric Food Chem 53: 7649-7653

[13] Kumar S, Singh AK, Kumar M, Yadav SK, Chauhan JS, Kumar PR (2003) Standardization of near infrared reflectance spectroscopy (NIRS) for determination of seed oil and protein contents in rape seed-mustard. J Food Sci Techn 40: 306-309 
[14] Velasco L, Goffman FD, Becker HC (1999) Development of calibration equations to predict oil content and fatty acid composition in Brassicaceae germplasm by near-infrared reflectance spectroscopy. J Am Oil Chem Soc 76, 25-30

[15] Siemens BJ, Daun JK (2005) Determination of the fatty acid composition of canola, flax, and solin by near-infrared spectroscopy. J Am Oil Chem Soc 82, 153-157

[16] IUPAC (1992) Oils and Fats. In: Dieffenbacher A and Pocklington WD (eds) Standard Methods for the Analysis of Oils, Fats and Derivatives. Blackwell Scientific Publications, Oxford, methods 2.301 and 2.303

[17] AOCS (1997) Oil. In: Firestone D (ed) Official Methods and Recommended Practices of the American Oil Chemists' Society, 5th edn., AOCS Press, Champaign, method Ba 3-38

[18] Wold S, Martens H, Wold H (1983) The Multivariate Calibration Problem in Chemistry solved by the PLS method. In: Ruhe A and Kågstrøm B (eds) Lecture notes in mathematics, Springer Verlag, Heidelberg, pp 286-293

[19] Williams P, Sobering D (1996) How do we do it: a brief summary of the methods we use in developing near infrared calibrations. In: Williams D and P (eds) Near Infrared Spectroscopy: The Future Waves. AMC , Ed AMC Davies and Phil Williams, Montreal, pp 185-189

[20] Ayerdi A, Farkasa E, Bergera M, Labaletteb, F, Centisc, S, Daydéa, J, Calmona, A (2007) Determination of tocopherols and phytosterols in sunflower seeds by NIR spectrometry. Eur J Lipid Sci Technol 109, 525-530 


\section{Legends}

Figure 1. Example of argan seed spectra acquired with the Labspec. Savitzky-Golay second derivative followed by standard normal variate transformation and absorbance data: (a) $1 / R$, inverse of reflectance data; (b) D2, second derivative. SNV, standard normal variate

Figure 2. Calibration with the Luminar of moisture prediction (a) and oil content prediction (b)

Figure 3. Prediction with the Labspec of: oleic acid (a); linoleic acid (b); palmitic acid (c); stearic 5 acid (d); U/S relation prediction (e) and OSI prediction (f) 

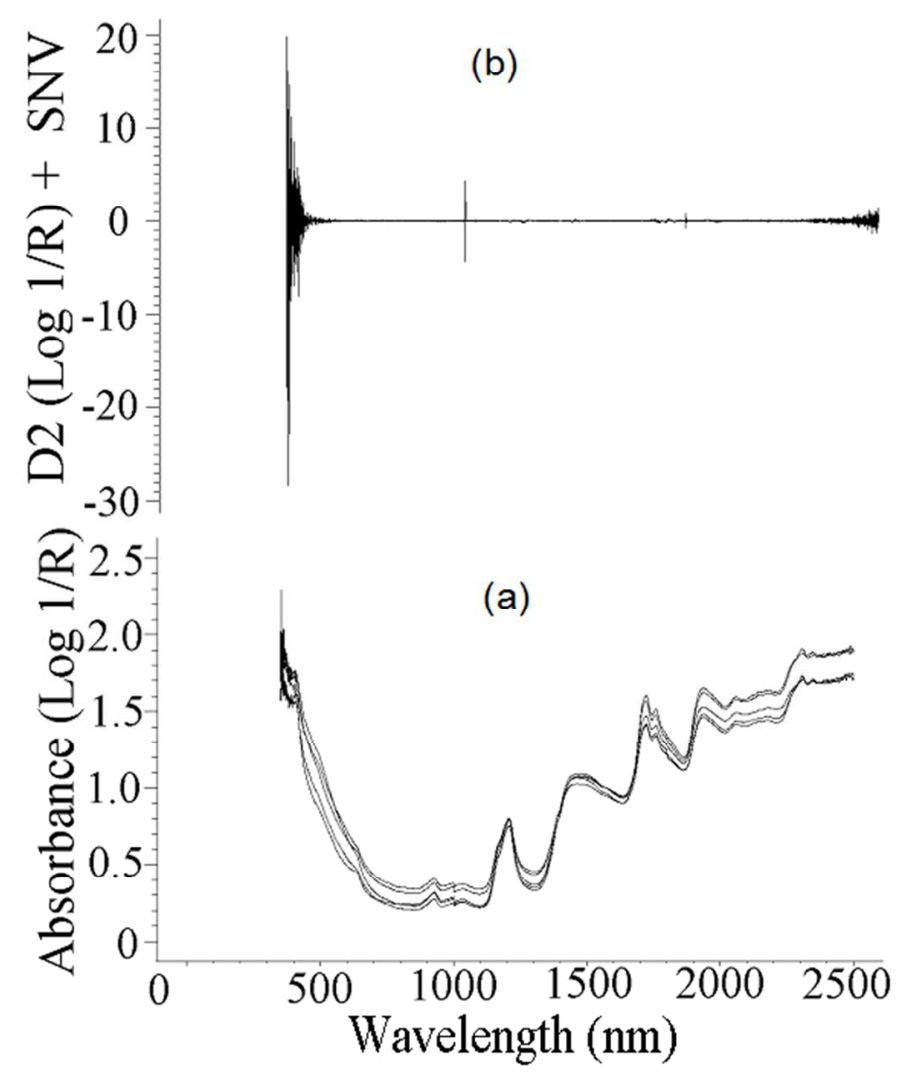

$301 \times 340 \mathrm{~mm}(72 \times 72$ DPI $)$ 

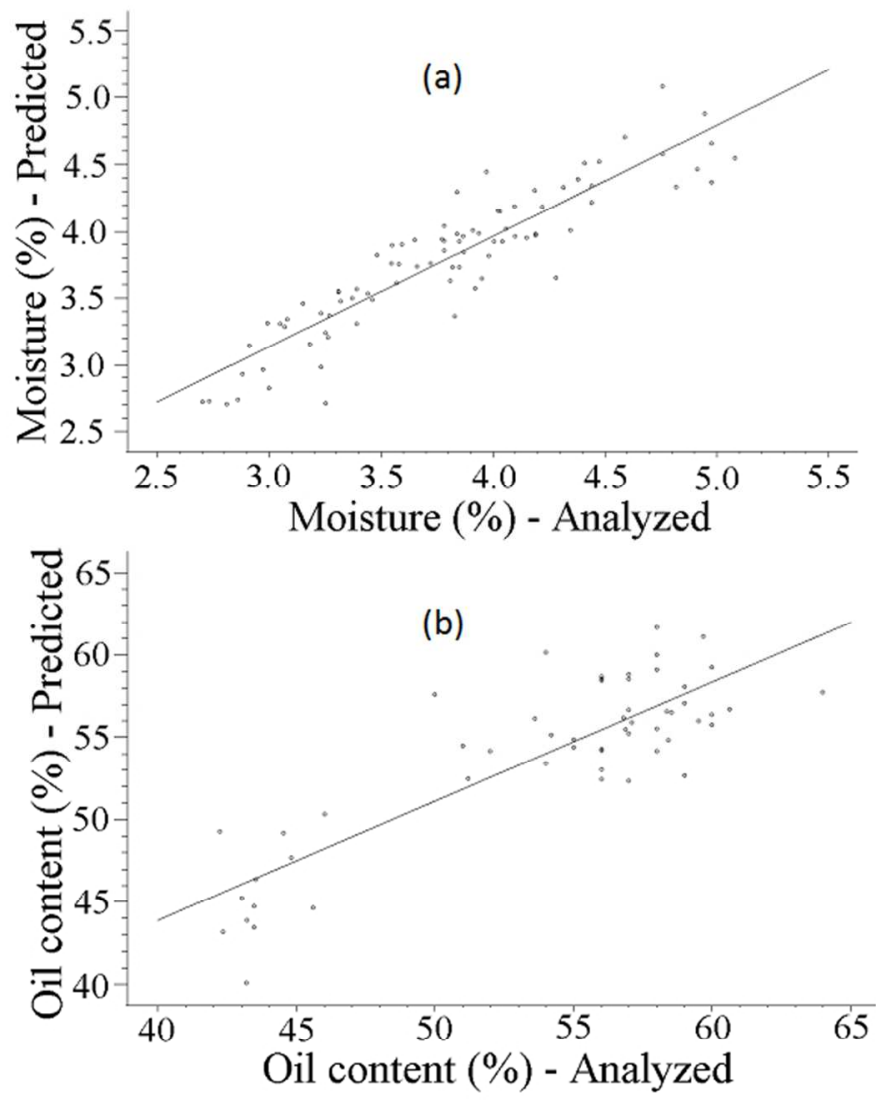

$303 \times 354 \mathrm{~mm}(72 \times 72$ DPI $)$ 

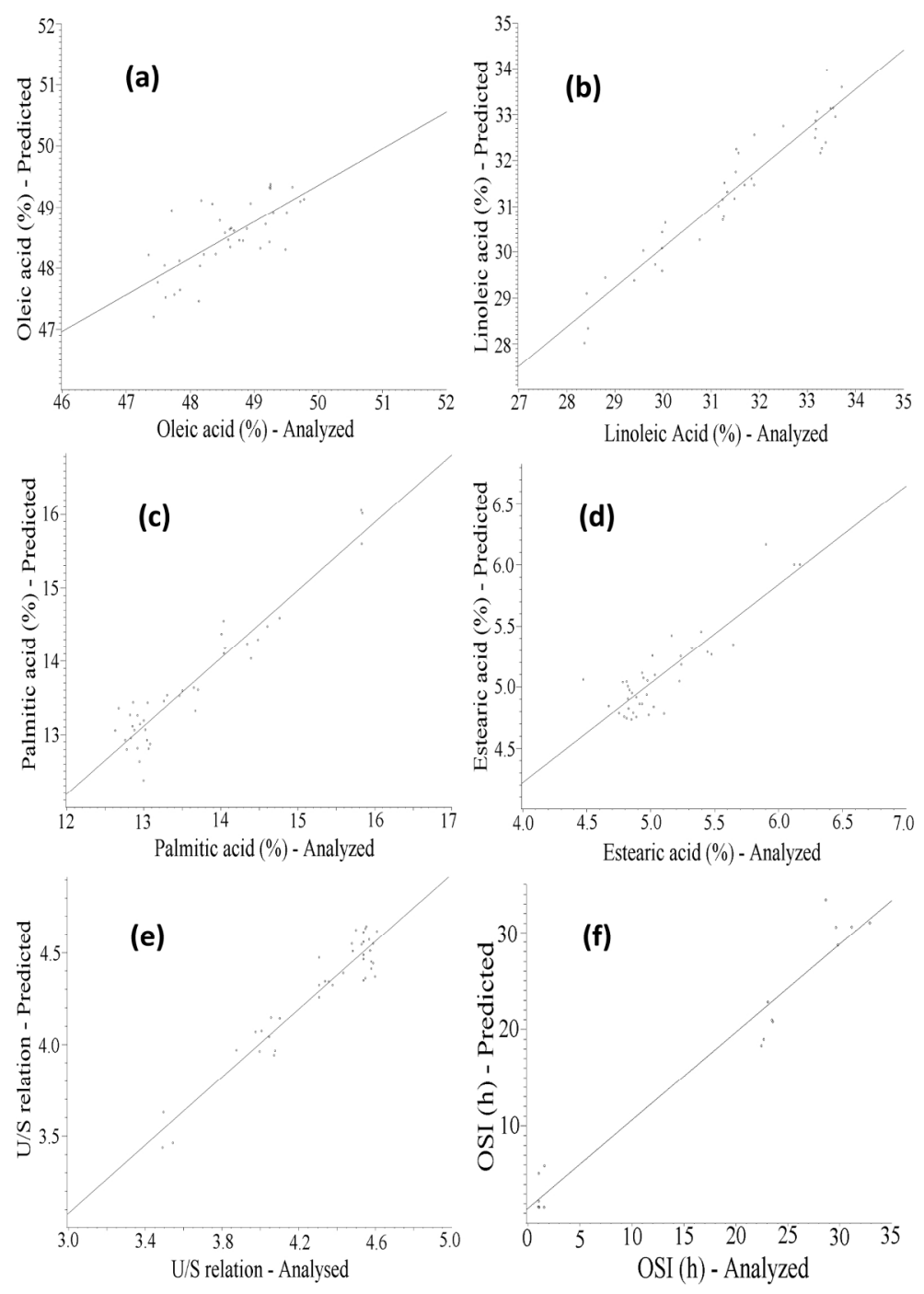

43

44

45

46

47

48

49

50

51

52

53

54

55

56

57

58

59

60

$153 \times 216 \mathrm{~mm}(300 \times 300 \mathrm{DPI})$ 
Table 1. Wavelengths selected for calibration with the Labspec

\begin{tabular}{ll}
\hline & Wavelengths selected for calibration $(\mathbf{n m})$ \\
\hline $\begin{array}{c}\text { oleic acid } \\
\text { linoleic acid }\end{array}$ & \multicolumn{1}{c}{$350-2500$} \\
& $350-2500$ \\
palmitic acid & $350-381,400-536,576-610,614-624,640-648,673-813,895-$ \\
& $1032,1220-1233,1393-1497,1531-1689,1801-1891,1904-$ \\
& $1969,2075-2117,2298-2318,2359-2500$ \\
& $350-384,388-392,396-542,568-629,638-652,669-851,887-$ \\
stearic acid & $1052,1156-1169,1178-1201,1213-1265,1271-1338,1356-$ \\
& $1497,1517-1701,1765-1893,1900-1991,2066-2142,2177-$ \\
& $2184,2225-2244,2295-2323,2355-2500$ \\
U/S & $350-540,560-652,663-858,884-1034,1040-1046,1141-$ \\
& $1186,1192-1136,1395-1497,1525-1701,1723-1730,1765-$ \\
& $1893,1899-1998,2057-2193,2223-2245,2296-2321,2352-$ \\
\hline U, major unsaturated fatty acids; S, major saturated fatty acids
\end{tabular}

20

21

22

23

24

25

26

27

28

29

30

31

32

33

34

35

36

37

38

39

40

41

42

43

44

45

46

47

48

49

50

51

52

53

54

55

56

57

58

59

60 
Table 2. Statistical data of the calibration and validation sets using Labspec

\begin{tabular}{|c|c|c|c|c|c|c|}
\hline \multicolumn{7}{|c|}{ CALIBRATIONS } \\
\hline parameter & samples & range & $\sigma$ & $\bar{X}$ & $\mathrm{CV}$ & $\mathbf{R} / \bar{X}$ \\
\hline oleic acid & 105 & $46.8-49.73$ & 0.59 & 48.50 & 1.2 & 6.0 \\
\hline linoleic acid & 105 & $28.2-49.69$ & 1.79 & 31.17 & 5.7 & 68.9 \\
\hline palmitic acid & 105 & $12.5-16.19$ & 1.17 & 13.73 & 8.5 & 26.9 \\
\hline estearic acid & 105 & $4.48-6.29$ & 0.49 & 5.18 & 9.5 & 34.9 \\
\hline $\mathrm{U} / \mathrm{S}$ & 105 & $3.40-5.02$ & 0.41 & 4.25 & 9.6 & 38.1 \\
\hline \multicolumn{7}{|c|}{ VALIDATIONS } \\
\hline parameter & samples & range & $\boldsymbol{\sigma}$ & $\bar{X}$ & $\mathrm{CV}$ & $\mathbf{R} / \bar{X}$ \\
\hline oleic acid & 27 & $46.2-49.6$ & 0.73 & 48.49 & 1.5 & 7.0 \\
\hline linoleic acid & 27 & $28.5-34.2$ & 1.87 & 31.23 & 6.0 & 18.3 \\
\hline palmitic acid & 27 & $12.4-16.0$ & 1.13 & 13.69 & 8.3 & 26.3 \\
\hline estearic acid & 27 & $4.63-6.13$ & 0.48 & 5.16 & 9.3 & 29.1 \\
\hline $\mathrm{U} / \mathrm{S}$ & 27 & $3.49-4.61$ & 0.32 & 4.31 & 7.42 & 26.0 \\
\hline
\end{tabular}


Table 3. Statistical coefficients for the models of the intact argan seeds

\begin{tabular}{|c|c|c|c|c|c|c|c|c|c|c|c|c|c|c|}
\hline \multicolumn{8}{|c|}{ Labspec } & \multicolumn{7}{|c|}{ Luminar 5030} \\
\hline & \multicolumn{3}{|c|}{$\begin{array}{c}\text { Calibration } \\
(\mathrm{N}=105)^{*}\end{array}$} & \multicolumn{4}{|c|}{$\begin{array}{c}\text { Validation } \\
(\mathrm{N}=27)\end{array}$} & \multicolumn{3}{|c|}{$\begin{array}{c}\text { Calibration } \\
(\mathrm{N}=105)\end{array}$} & \multicolumn{4}{|c|}{$\begin{array}{c}\text { Validation } \\
(\mathrm{N}=35)\end{array}$} \\
\hline & $\mathbf{R}_{\mathbf{C}}$ & $\mathbf{R}_{\mathbf{C V}}$ & SEC & RMSEP & RPD & $\mathrm{CV}$ & $\mathbf{R} / \bar{X}$ & $\mathbf{R}_{\mathbf{C}}$ & $\mathbf{R}_{\mathrm{CV}}$ & SEC & RMSEP & RPD & $\mathrm{CV}$ & $\mathbf{R} / \bar{X}$ \\
\hline oleic acid & 0.89 & 0.86 & 0.27 & 0.35 & 2.07 & 1.5 & 7.01 & 0.67 & 0.57 & 0.49 & - & - & 1.0 & 4.86 \\
\hline linole & 0.98 & 0.97 & 0.39 & 0.39 & 4.69 & 6.0 & 18.25 & 0.90 & 0.77 & 0.71 & 0.93 & 1.75 & 5.6 & 15.42 \\
\hline palmi & 0.96 & 0.95 & 0.32 & 0.34 & 3.32 & 8.3 & 26.29 & 0.89 & 0.87 & 0.46 & 0.67 & 1.52 & 7.2 & 21.10 \\
\hline stearic a & 0.96 & 0.95 & 0.31 & 0.17 & 2.78 & 9.3 & 29.07 & 0.84 & 0.81 & 0.25 & 0.26 & 1.72 & 8.7 & 26.44 \\
\hline $\mathrm{U} / \mathrm{S}$ & 0.97 & 0.96 & 0.10 & 0.11 & 3.64 & 7.42 & 25.99 & 0.90 & 0.87 & 0.16 & 0.21 & 1.74 & 8.8 & 23.91 \\
\hline OSI & 0.99 & 0.98 & 1.94 & 2.60 & 4.88 & 0.8 & 96.5 & & & & & & & \\
\hline
\end{tabular}

*OSI: calibration $\mathrm{N}=50$ and validation $\mathrm{N}=16 . \mathrm{N}$, set size; $\mathrm{RC}$, coefficient of calibration; $\mathrm{RCV}$, coefficient of cross validation; SEC, standard error of the calibration; RMSEP, root mean squared error of prediction; RPD, residual predictive deviation; $C V$, coefficient of variation; $\bar{X}$, mean; $R$, relative range. 
Table 4. Evolution of majority fatty acids (\%) in the forced oxidation assay

\begin{tabular}{lcccc}
\hline & \multicolumn{3}{c}{$\bar{X} \pm \sigma$} \\
days $\left[\mathbf{n}^{\mathbf{o}}\right.$ samples] \\
parameter & $\mathbf{2 ~ [ 6 2 ]}$ & $\mathbf{4}[\mathbf{6 2}]$ & $\mathbf{1 2}[\mathbf{4 9 ]}$ & $\mathbf{2 4}[\mathbf{4 7 ]}$ \\
\hline oleic acid & $47.8 \pm 0.39^{\mathrm{a}}$ & $48.7 \pm 0.33^{\mathrm{b}}$ & $49.2 \pm 0.33^{\mathrm{c}}$ & $48.8 \pm 0.49^{\mathrm{b}}$ \\
linoleic acid & $33.3 \pm 0.35^{\mathrm{a}}$ & $31.8 \pm 0.48^{\mathrm{b}}$ & $30.6 \pm 0.67^{\mathrm{c}}$ & $29.0 \pm 0.66^{\mathrm{d}}$ \\
palmitic acid & $12.9 \pm 0.17^{\mathrm{a}}$ & $13.0 \pm 0.20^{\mathrm{a}}$ & $13.9 \pm 0.40^{\mathrm{b}}$ & $15.2 \pm 0.72^{\mathrm{c}}$ \\
stearic acid & $4.9 \pm 0.12^{\mathrm{a}}$ & $4.9 \pm 0.16^{\mathrm{a}}$ & $5.1 \pm 0.25^{\mathrm{b}}$ & $5.8 \pm 0.35^{\mathrm{c}}$ \\
$\mathrm{U} / \mathrm{S}$ & $4.6 \pm 0.04^{\mathrm{a}}$ & $4.5 \pm 0.06^{\mathrm{b}}$ & $4.2 \pm 0.14^{\mathrm{c}}$ & $3.7 \pm 0.23^{\mathrm{d}}$ \\
\hline $\bar{X}$, mean; $\sigma$, standard deviation; $\mathrm{U}$, major unsaturated fatty acids; $\mathrm{S}$, major saturated fatty acids Data sharing the same letter in rows (a-d) \\
were not significantly different $(\mathrm{P} \leq 0.001)$.
\end{tabular}


Table 5. Statistical coefficients of the models (a) and statistical data (b) of argan seed moisture and oil content using Luminar 5030

\section{(b) STATISTICAL DATA}

\begin{tabular}{lcccccc}
\hline \multicolumn{7}{c}{ CALIBRATIONS } \\
parameter & $\mathbf{N}$ & range & $\mathbf{\sigma}$ & $\bar{X}$ & $\mathbf{C V}$ & $\mathbf{R} / \bar{X}$ \\
\hline moisture & 85 & $2.91-5.51$ & 0.56 & 3.87 & 0.1 & 67.2 \\
\hline oil content & 55 & $42.22-64.00$ & 5.98 & 53.98 & 0.1 & 40.4 \\
\hline \multicolumn{7}{c}{ VALIDATIONS } \\
parameter & $\mathbf{N}$ & range & $\mathbf{\sigma}$ & $\bar{X}$ & $\mathbf{C V}$ & $\mathbf{R} / \mathbf{M}$ \\
\hline moisture & 20 & $2.57-4.82$ & 0.63 & 3.74 & 0.2 & 60.2 \\
\hline oil content & 14 & $43.19-60.62$ & 5.76 & 53.38 & 0.1 & 32.7 \\
\hline
\end{tabular}

(a) STATISTICAL COEFFICIENTS

\begin{tabular}{llccccc}
\hline parameter & $\mathbf{N}$ & $\mathbf{R}_{\mathbf{C}}$ & $\mathbf{R}_{\mathbf{C V}}$ & SEC & RMSEP & RPD \\
\hline moisture & 85 & 0.91 & 0.69 & 0.24 & 0.32 & 2.13 \\
\hline oil content & 52 & 0.88 & 0.88 & 2.73 & 2.62 & 2.44 \\
\hline
\end{tabular}

$\mathrm{N}$, set size; $\sigma$, standard deviation; $\bar{X}$, mean; $\mathrm{CV}$, coefficient of variation; $\mathrm{R}$, relative range; $\mathrm{R}_{\mathrm{C}}$, coefficient of calibration; $\mathrm{R}_{\mathrm{CV}}$, coefficient of cross validation; SEC, standard error of the calibration; RMSEP, root mean squared error of prediction; RPD, residual predictive deviation 